\title{
Anti-jamming approach based on radar transmitted waveform matching
}

\author{
Xiaoying Chen and Baixiao Chen ${ }^{*}$ (D)
}

${ }^{*}$ Correspondence:

bxchen@xidian.edu.cn

National Laboratory of Radar Signal

Processing, Xidian University, Taibai

South Road, 710071 Xi'an, China

\begin{abstract}
This study proposes a novel approach to suppress noise jamming and smart jamming. The traditional method of using auxiliary channels to cancel interference requires pure interference samples to calculate weights, which is almost impossible for pulsed interference signals. In this work, to avoid the difficulty of choosing suitable interference samples, we construct the parameterized expected signal according to the time-delay relation between target reflecting echo and transmitted signal. The objective function is established in the form of the minimum mean square error between the recovered signal and the expected signal. The optimization problem is solved by an alternating iteration method. Simulation results demonstrate that the proposed method achieves excellent performance for suppressing noise jamming and smart jamming and is not sensitive to signal-to-noise ratio and jamming-to-noise ratio. The processing results of the measured data show that the method has a certain practical application value.
\end{abstract}

Keywords: Anti-jamming, Noise jamming, Smart jamming, Optimization

\section{Introduction}

The complex electromagnetic interference environment seriously threatens the survival and performance of the radar system [1].

Noise jamming (NJ) is a kind of noise-like signal, which greatly reduces the signal-tonoise ratio (SNR) on hostile radar receivers, making it difficult to detect valuable signals or generate errors [2]. Target detection in the background of noise interference [3-8] is a basic way to reduce its masking impact. If the interference is much powerful, the receiver will be saturated, and the valuable signal will be completely submerged. It is not very meaningful to apply the detection algorithms in this scenario. Some technical measures have been proposed to eliminate noise jamming in [9-11]. However, these methods usually rely on mathematical models of interference. Due to the noise-like characteristics of the interference, a slight deviation between the actual interference signal and the ideal model will degrade the performance of the anti-jamming algorithm and even fail. These methods will be limited in practical situations. Besides, the idea of blind source separation is used to separate target signal and interference [12,13]. The methods based on blind source separation have low requirements on the prior information and improve anti-interference effectiveness to a certain extent. Due to the inherent amplitude and

(c) The Author(s). 2021 Open Access This article is licensed under a Creative Commons Attribution 4.0 International License, which permits use, sharing, adaptation, distribution and reproduction in any medium or format, as long as you give appropriate credit to the original author(s) and the source, provide a link to the Creative Commons licence, and indicate if changes were made. The images or other third party material in this article are included in the article's Creative Commons licence, unless indicated otherwise in a credit line to the material. If material is not included in the article's Creative Commons licence and your intended use is not permitted by statutory regulation or exceeds the permitted use, you will need to obtain permission directly from the copyright holder. To view a copy of this licence, visit http://creativecommons.org/licenses/by/4.0/. 
ordering uncertainty of the algorithm, it is necessary to further identify the separated signal properties, which is also the limitation of this series of methods.

Smart jamming [2] is a kind of interference with excellent application prospects. This type of interference can obtain pulse compression (PC) gain and effectively use signal power. There are several methods to suppress smart noise jamming, like fractional domain filtering [14], smeared spectrum [15], atomic decomposition [16] and blind separation [17]. These methods usually rely on prior assumptions on interference delay, frequency shift and other information heavily. From this point, we hope to find a method with fewer prior assumptions, which is more in line with the randomness of the interference scene and can deal with a variety of modulation sequences.

Multi-channel data provides more freedom for adaptive processing. The correlation of multi-channel data can be used to further improve the output SNR and achieve better detection performance [18]. Combining the main channel and the auxiliary channel for filtering is a very effective method to deal with various interferences [17, 19]. Similarly, we propose a novel approach to suppress noise jamming and smart jamming in which two aliased signals are weighted to recover the target. Interference suppression is transformed into the problem of target signal recovery based on radar transmitted waveform matching. The optimization goal is to minimize the mean square error between the recovered signal and the expected signal. The expected signal expression is constructed according to the time-delay relation of target echo and transmitting waveform and is updated continuously during iteration. The alternating iteration method is used to solve the optimization problem and recover the target echo.

Compared with existing methods, the proposed algorithm does not require pure interference signal samples, making it easy to implement. The proposed algorithm can be used to deal with the case of NJ and smart jamming. Flexible modulation of interference parameters does not affect the suppression effect. These advantages make it more suitable for the practical application scenario. Simulation and measured data are used to verify the correctness of the theoretical analyses.

The paper is organized as follows. Section 2 introduces the model of two receiving channels. Section 3 introduces NJ and smart jamming and proposes the algorithm to suppress these two kinds of jamming. In Section 4, simulation results and performance analysis are given. An example of measured data is provided to illustrate the practicability of the proposed method. Finally, Section 5 concludes the paper.

Notations: $(\cdot)^{\mathrm{T}}$ denotes transpose operation, $(\cdot)^{*}$ denotes complex conjugate operation, $(\cdot)^{\mathrm{H}}$ denotes conjugate transpose operation, $\otimes$ denotes the convolution operation, $\odot$ denotes the Hadamard product. $\|\cdot\|$ denotes the Euclidean norm.

\section{Channel model}

Radar should tap as much potential as possible based on existing hardware resources to improve the adaptability and survivability in different work scenarios. The polarization difference between the main antenna and the auxiliary antenna is used to realize two independent observation channels. The polarization mode between the auxiliary antenna and the main antenna is orthogonal.

Under the horizontal and vertical polarization basis, the target signal and jamming signal in the main channel can be expressed as

$$
\mathbf{v}_{\mathbf{x}}=\mathbf{S}_{\mathrm{p}} \mathbf{h}_{\mathrm{m}} A_{\mathrm{m}, \mathrm{x}} x(t)
$$




$$
\mathbf{v}_{\mathbf{j}}=\mathbf{h}_{\mathbf{j}} A_{\mathrm{m}, j} j(t)
$$

where $x(t), j(t)$ represent the target scattering waveform and the interference waveform with normalized amplitude $1 ; \mathbf{h}_{\mathrm{m}}, \mathbf{h}_{\mathrm{j}}$ denote the polarization vector of the radar main antenna and the jammer transmitting antenna; $\mathbf{S}_{\mathrm{p}}$ is the polarization scattering matrix of the radar target at the current attitude and current frequency. $A_{\mathrm{m}, \mathrm{x}}, A_{\mathrm{m}, \mathrm{j}}$ represent target signal amplitude and jamming amplitude in the main channel considering transmission power, antenna gain, working wavelength, radar cross-section (RCS) and other factors except for the polarization information.

The mixed signal received by the main channel is expressed as:

$$
y_{1}(t)=\mathbf{h}_{\mathrm{m}}^{\mathrm{T}} \mathbf{v}_{\mathbf{x}}+\mathbf{h}_{\mathrm{m}}^{\mathrm{T}} \mathbf{v}_{\mathbf{j}}+n_{1}(t)
$$

where $n_{1}(t)$ is the Gaussian white noise in the main channel.

Similarly, the polarization vector of the cross-polarization auxiliary antenna is $\mathbf{h}_{\mathrm{a}}$. The target signal and jamming signal of the auxiliary channel is decomposed under the polarization basis and expressed as:

$$
\begin{aligned}
& \mathbf{u}_{\mathbf{x}}=\mathbf{S}_{\mathrm{p}} \mathbf{h}_{\mathrm{m}} A_{\mathrm{a}, \mathrm{x}} x(t) \\
& \mathbf{u}_{\mathbf{j}}=\mathbf{h}_{\mathbf{j}} A_{\mathrm{a}, \mathrm{j}} j(t)
\end{aligned}
$$

where $A_{\mathrm{a}, \mathrm{x}}, A_{\mathrm{a}, \mathrm{j}}$ denote target signal amplitude and jamming amplitude in the auxiliary channel.

The mixed signal received by the auxiliary channel is expressed as

$$
y_{2}(t)=\mathbf{h}_{\mathrm{a}}^{\mathrm{T}} \mathbf{u}_{\mathbf{x}}+\mathbf{h}_{\mathrm{a}}^{\mathrm{T}} \mathbf{u}_{\mathbf{j}}+n_{2}(t)
$$

where $n_{2}(t)$ is the Gaussian white noise in the auxiliary channel.

\section{Suppression method}

Interference suppression can be understood as recovering the target echo from the aliased signal or extracting target information through various technical means. The target reflects the radar transmitted signal, and the waveform of the echo signal is consistent with the radar transmitted signal, with time-delay and Doppler information modulation. In this section, this feature of the target echo is used to model the interference suppression problem as finding the minimum mean square error (MMSE) between the recovered signal and the expected signal. An optimized objective function is established.

\subsection{Signal model of jamming}

In this section, the signal models of NJ, smart jamming are given. NJ has similar characteristics with radar internal noise and completely covers the target echo in the time domain and frequency domain. The implementation of $\mathrm{NJ}$ does not require accurate information about the radar working mode.

This article takes radio frequency (RF) noise jamming as an example. RF noise jamming is a narrow-band Gaussian random process, usually generated by filtering and amplifying low-power noise. The interference signal can be expressed as

$$
J_{n}(t)=U_{n}(t) \exp \left[\mathrm{j}\left(w_{\mathrm{j}} t+\varphi(t)\right)\right]
$$


where, $J_{n}(t)$ follows the normal distribution, and its envelope function $U_{n}(t)$ follows the Rayleigh distribution; the phase function $\varphi(t)$ follows the uniform distribution of $[0,2 \pi]$, and is independent of $U_{n}(t)$. The carrier frequency $w_{\mathrm{j}}$ is constant and much larger than the spectral width of $J_{n}(t)$.

In addition to the RF noise jamming, there are other common NJ forms: noise amplitude modulation, noise frequency modulation, noise phase modulation, etc. The proposed anti-jamming method is effective for all of these interference forms, and no specific distinction is made here.

Smart jamming is generated by convolving the radar transmitted signal copy stored in the digital radio frequency memory (DRFM) with a random pulse train, giving the jamming signal the same PC gain as the real target echo and making the jamming power utilized fully. It has both deception and blanket effects.

Assume the transmitted radar signal is $s(t)$. The jammer intercepts the radar signal, and the sample signal is formed after a series of processing [2]. Then, the sample signal is convolved with a random pulse train to obtain a new convolution sequence. The random pulse train is expressed as:

$$
m(t)=\sum_{k=1}^{K} A_{k} \delta(t-k \Delta t)
$$

where $K$ represents the total length of the random pulse train, $\Delta t$ is the sampling time interval, $\delta(t)$ represents the Dirac delta function, and $A_{k}$ is the amplitude of $k$ th shock signal.

The convolution of the random pulse train and the intercepted radar signal yields:

$$
\begin{aligned}
J_{c}(t)=s(t) \otimes m(t) & =s(t) \otimes \sum_{k=1}^{K} A_{k} \delta(t-k \Delta t) \\
& =\sum_{k=1}^{K} A_{k} s(t-k \Delta t) .
\end{aligned}
$$

Smart noise interference has similar characteristics to target echo. It is a kind of coherent jamming that matches the radar transmitted waveform and can effectively utilize the PC gain. Compared with the traditional noise modulation interference and other non-coherent interferences, smart interference with the same power can form a better effect.

The parameters of the random pulse train determine that smart jamming will have a blanket or deception effect. When the sampling interval of the modulation sequence used by the jammer is less than or equal to the radar sampling interval, convolution noise jamming $(\mathrm{CNJ})$ is formed, and such interference generally exerts a blanket effect. On the contrary, when it is far beyond the radar sampling interval, a train of false targets is produced at the processing output of the victim radar. It is called dense false target jamming (DFTJ).

\subsection{Objective function}

Assume radar transmits a pulsed linear frequency modulated (LFM) signal. Since the echo signal is processed after being down-converted, the effect of the carrier frequency can be ignored. The complex envelope of transmitted signal can be expressed as: 


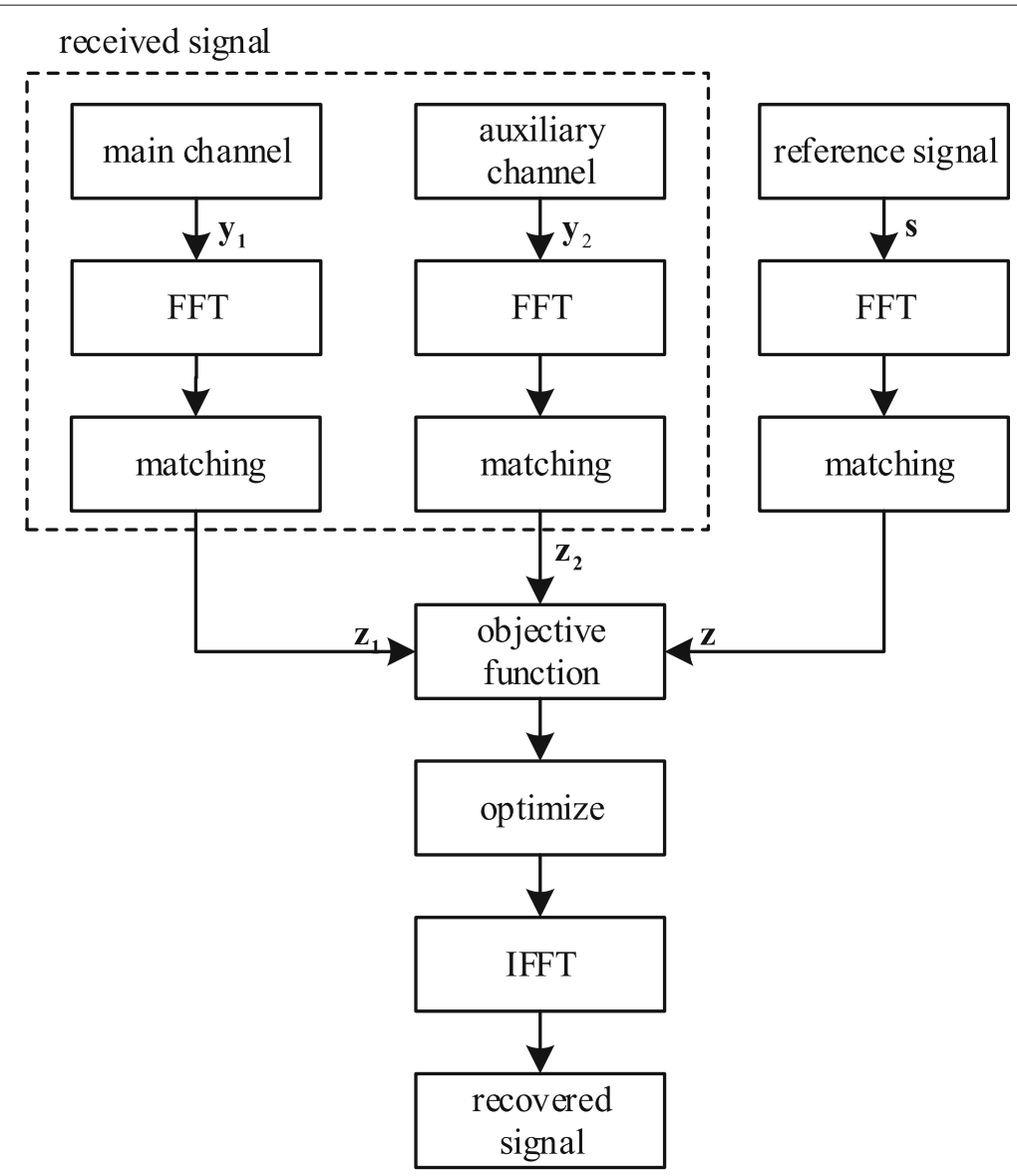

Fig. 1 Flowchart of the proposed algorithm

$$
s(t)=\operatorname{rect}\left(\frac{t}{T_{\mathrm{p}}}\right) \exp \left(\mathrm{j} \pi \mu t^{2}\right), t \in\left(-\frac{T_{\mathrm{r}}}{2}, \frac{T_{\mathrm{r}}}{2}\right)
$$

where $T_{\mathrm{r}}$ is the pulse repetition interval (PRI), $T_{\mathrm{p}}$ is the pulse duration, $\mu=B / T_{\mathrm{p}}$ is the chirp rate, and $B$ is the bandwidth of the frequency modulation, which generally satisfy $B T_{\mathrm{p}}>>1$. The function $\operatorname{rect}(t)$ has the following form

$$
\operatorname{rect}\left(\frac{t}{T_{\mathrm{P}}}\right)=\left\{\begin{array}{l}
1|t| \leq T_{\mathrm{P}} / 2 \\
0|t|>T_{\mathrm{P}} / 2
\end{array} .\right.
$$

Suppose a target with distance $R$ and velocity $v$, the target echo in $i$ th pulse can be expressed as:

$$
\begin{aligned}
x_{i}(t) & =\alpha_{i} s(t-\tau) \exp \left(\mathrm{j} 2 \pi f_{\mathrm{d}}\left(t+(i-1) T_{\mathrm{r}}-\tau\right)\right) \\
& =\tilde{\alpha}_{i} s(t-\tau) \exp \left(\mathrm{j} 2 \pi f_{\mathrm{d}} t\right)
\end{aligned}
$$

where $i=1,2, \ldots, M, M$ is the pulse number in one correlation processing interval (CPI). $\tau=2 R_{0} / c$ is the delay corresponding to the distance of the target, $f_{\mathrm{d}}=\frac{2 v}{\lambda}$ is the Doppler frequency corresponding to the velocity, $\lambda$ denotes wavelength, $\alpha_{i}$ represents target signal amplitude in $i$ th PRT. $\widetilde{\alpha_{i}}=\alpha_{i} \exp \left(\mathrm{j} 2 \pi f_{\mathrm{d}}\left((i-1) T_{\mathrm{r}}-\tau\right)\right)$. For the target echo in $i$ th pulse, the Doppler information of slow-time domain is contained in complex amplitude 


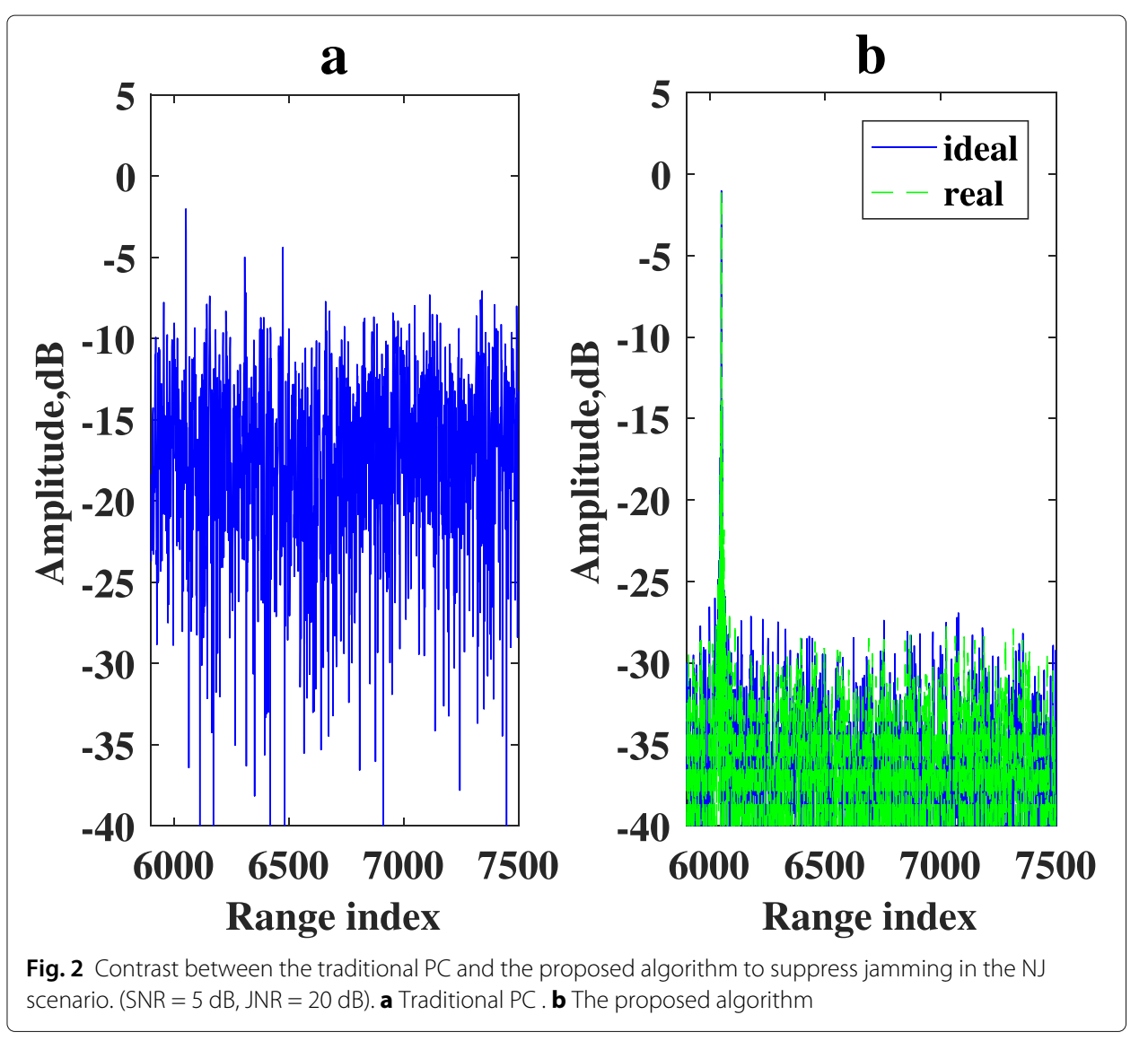

$\widetilde{\alpha_{i}}$. The Doppler information of fast-time domain is contained in the exponential term $\exp \left(\mathrm{j} 2 \pi f_{\mathrm{d}} t\right)$ which will be ignored in the following derivation.

For the sake of brevity, we omit the sign $i$. Radar receives the mixed signal of target echo and jamming and an inevitable noise. Design the filter to appropriately weigh the received two-way signal (amplitude and phase weighting) so that the output result of the filter contains the target echo as much as possible. The filter coefficient is $a$ and $b . c$ represents the amplitude information of the target echo. This is equivalent to solving the following optimization problem:

$$
\min \left\|a y_{1}(t)+b y_{2}(t)-c x(t)\right\|^{2}
$$

Before the interference suppression processing is performed on the received signal, the target echo cannot be known, and the above optimization problem cannot be directly solved. This section uses the characteristics of the target echo to transform the form of the objective function and obtain a new optimization problem that is easy to solve.

The signal is transformed to the frequency domain and expressed as a function of frequency. The frequency spectrum of the target signal can be written as:

$$
X\left(e^{\mathrm{j} w}\right)=S\left(e^{\mathrm{j} w}\right) \exp (-\mathrm{j} w \tau)
$$

For smart jamming, the frequency spectrum has the following form:

$$
J\left(e^{\mathrm{j} w}\right)=S\left(e^{\mathrm{j} w}\right) \sum_{i=1}^{K} \exp \left(-\mathrm{j} w \tau_{i}\right)
$$




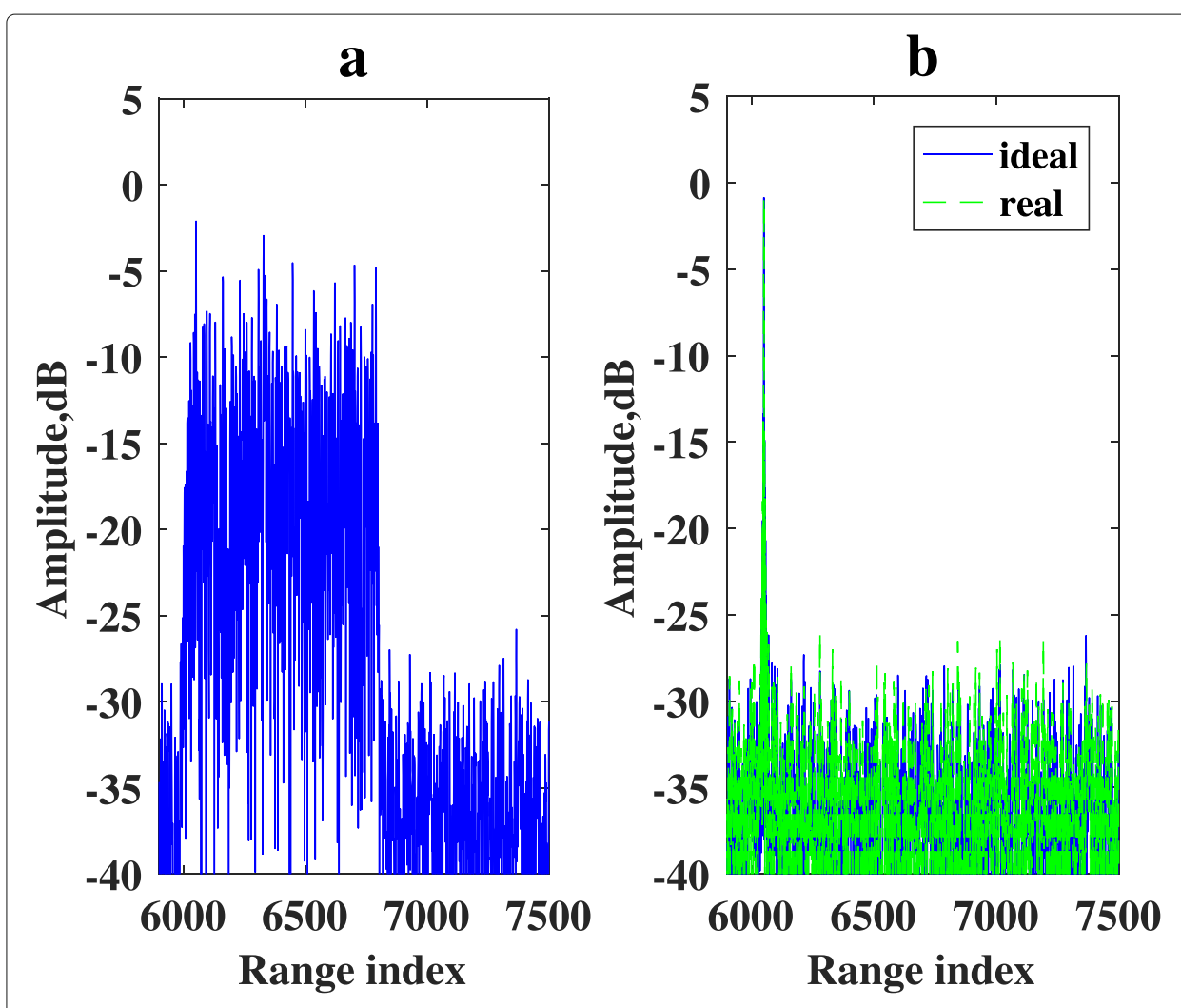

Fig. 3 Contrast between the traditional PC and the proposed algorithm to suppress jamming in the CNJ scenario. ( $S N R=5 \mathrm{~dB}, J N R=15 \mathrm{~dB}$ ). a Traditional PC. b The proposed algorithm

Define a characteristic factor in the form of the ratio of the frequency spectrum to characterize the matching relationship between two signals, for target echo,

$$
\left|\frac{X\left(e^{j w}\right)}{S\left(e^{j w}\right)}\right|=\left|e^{-j w \tau}\right|=1
$$

When $K \gg 1$, this feature of the smart jamming is

$$
\left|\frac{J\left(e^{\mathrm{j} w}\right)}{S\left(e^{\mathrm{j} w}\right)}\right|=\left|\sum_{i=1}^{K} \exp \left(-\mathrm{j} w \tau_{i}\right)\right| \neq 1
$$

Obviously, the unequal relationship between the characteristic factor and 1 is also valid for NJ. In the following derivation, we ignore noise component $n(t)$ for now and try to recover the target echo $x(t)$ from $y(t)$ based on the difference in the characteristic factor of interference and target echo.

The actual signal will be processed by discrete sampling, so the waveforms of (12) are separately rewritten as a finite-length sequence $\left\{x_{n}\right\}_{n=1}^{N}$ and $\left\{s_{n}\right\}_{n=1}^{N}$, expressed as $N \times 1$ vector $\mathbf{x}$ and $\mathbf{s}$, where $N$ represents the number of effective sampling points in a PRI.

Perform Fourier transform on the two sides of (12)

$$
\sum_{n=1}^{N} x(n) \mathrm{e}^{-\mathrm{j} w n}=e^{-\mathrm{j} w n_{0}} \sum_{n=1}^{N} s(n) \mathrm{e}^{-\mathrm{j} w n}
$$

where $n_{0}$ is the index of the range bin corresponding to the target delay, Let

$$
\mathbf{a}_{p}^{*}=\left[e^{-\mathrm{j} w_{p}} \cdots e^{-\mathrm{j} N w_{p}}\right]
$$




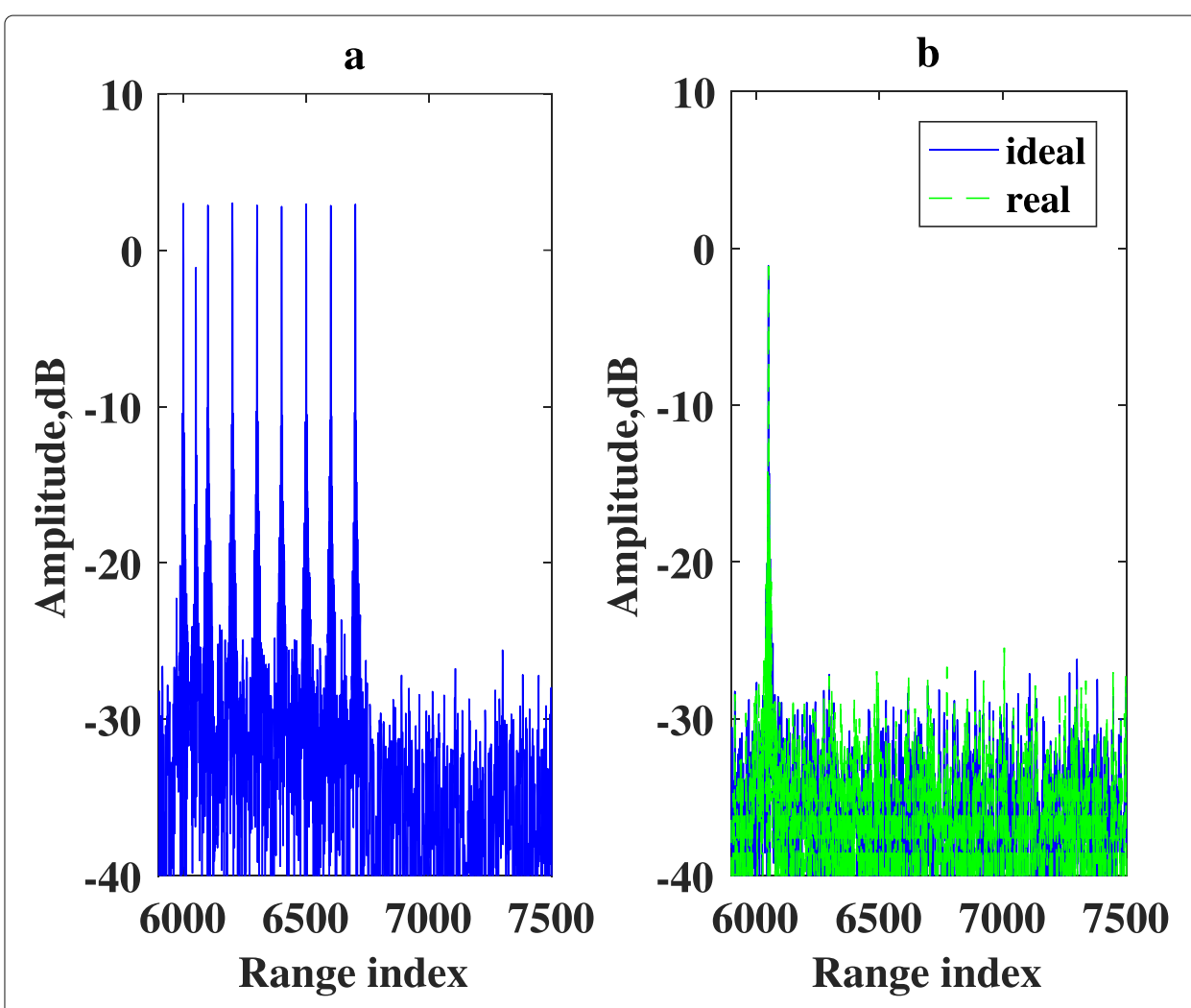

Fig. 4 Contrast between the traditional PC and the proposed algorithm to suppress jamming in the DFTJ scenario. (SNR $=5 \mathrm{~dB}, \mathrm{JNR}=15 \mathrm{~dB}$ ). a Traditional $\mathrm{PC}$. b The proposed algorithm

where $w_{p}$ are the following Fourier frequencies $w_{p}=\frac{2 \pi}{N} p, p=1, \cdots, N$

Let $\mathbf{A}^{*}$ be the following unitary $N \times N$ fast Fourier transform (FFT) matrix

$$
\mathbf{A}^{*}=\frac{1}{\sqrt{N}}\left[\begin{array}{l}
\mathbf{a}_{1}^{*} \\
\vdots \\
\mathbf{a}_{N}^{*}
\end{array}\right]
$$

and introduce an auxiliary vector $\mathbf{d},(18)$ is rewritten as:

$$
\mathbf{A}^{*} \mathbf{x}=\mathbf{A}^{*} \mathbf{s} \odot \mathbf{d}
$$

where $\mathbf{d}=c \mathbf{v}, \mathbf{v}$ has the following form

$$
\mathbf{v}=\left[e^{-\mathrm{j} w_{1} n_{0}}, e^{-\mathrm{j} w_{2} n_{0}}, \cdots, e^{-\mathrm{j} w_{N} n_{0}}\right]^{\mathrm{T}} .
$$

Multiply $\left(\mathbf{A}^{*} \mathbf{s}\right)^{*}$ on both sides of (21), which is equivalent to perform matching operation to LFM signal in frequency domain, i.e.,

$$
\left(\mathbf{A}^{*} \mathbf{x}\right) \odot\left(\mathbf{A}^{*} \mathbf{s}\right)^{*}=\left(\mathbf{A}^{*} \mathbf{s}\right) \odot\left(\mathbf{A}^{*} \mathbf{s}\right)^{*} \odot \mathbf{d} .
$$

The waveform $y_{1}(t)$ in the main channel can be expressed as $N \times 1$ vector $\mathbf{y}_{\mathbf{1}}$, and the waveform $y_{2}(t)$ in the cross-polarization auxiliary channel can be expressed as $N \times 1$ vector $\mathbf{y}_{2}$. Using the prior information, the target echo can be recovered from the two aliased signals. To facilitate the derivation of the formula, the time domain aliased signal is converted into the frequency domain. 


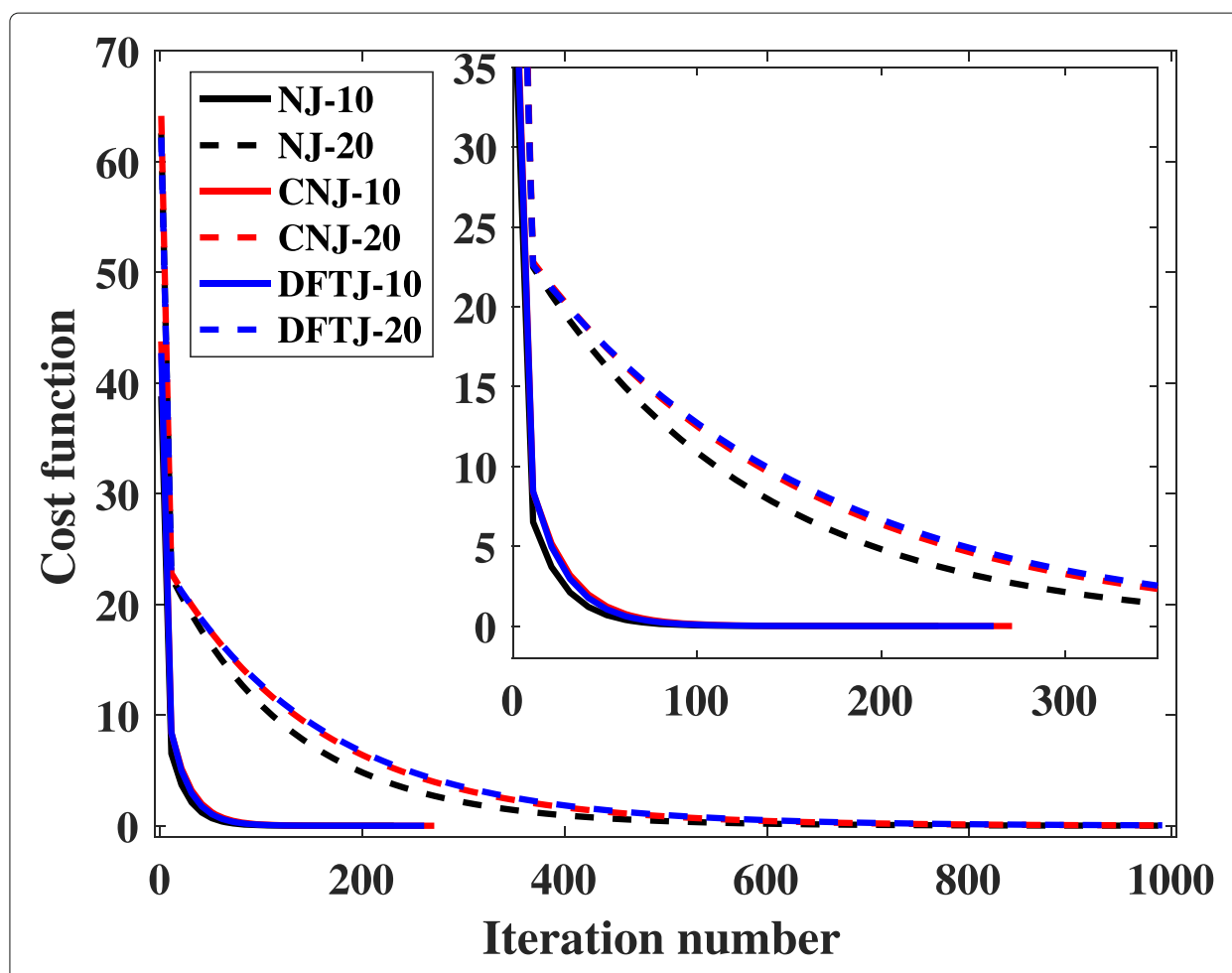

Fig. 5 Cost function in iteration process ( $S N R=5 \mathrm{~dB}, J N R=10 \mathrm{~dB}$ or $\mathrm{JNR}=20 \mathrm{~dB}$ )

Perform FFT and frequency-domain matching operation on the two mixed signals separately to obtain

$$
\begin{aligned}
& \mathbf{z}_{\mathbf{1}}=\left(\mathbf{A}^{*} \mathbf{y}_{\mathbf{1}}\right) \odot\left(\mathbf{A}^{*} \mathbf{s}\right)^{*} \\
& \mathbf{z}_{\mathbf{2}}=\left(\mathbf{A}^{*} \mathbf{y}_{\mathbf{2}}\right) \odot\left(\mathbf{A}^{*} \mathbf{s}\right)^{*}
\end{aligned}
$$

The radar transmitted signal is used as a reference signal. Perform FFT and frequencydomain matching operation on the reference signal in time domain to obtain the frequency-domain reference signal, that is

$$
\mathbf{z}=\left(\mathbf{A}^{*} \mathbf{s}\right) \odot\left(\mathbf{A}^{*} \mathbf{s}\right)^{*}
$$

A set of coefficients is used to weight the two frequency-domain aliased signals. According to (21), the expected signal is expressed as the Hadamard product of the reference signal spectrum and the auxiliary vector. The variable $c$ controls the amplitude scaling of the reference signal. The optimization function is expressed in the MMSE form between the recovered signal and the expected signal:

$$
O=\left\|a \mathbf{z}_{1}+b \mathbf{z}_{2}-c \mathbf{z} \odot \mathbf{v}\right\|^{2}
$$

where $a, b$ denote weight coefficient in the main channel and the auxiliary channel.

The traditional methods directly collect some interference samples from the rest area of the radar as the desired signal. The proposed method is significantly different from it, which takes the target echo as the desired signal. In this paper, the algorithm is derived based on the idea of transmitting waveform matching, and its core is to restore the target component without interference sample collection. 


\subsection{Optimization}

The objective function in (27) is the function of $a, b$, and auxiliary variable $c$ and auxiliary vector v. This is a multivariate optimization problem. Since the expected signal is not completely sure and needs to be obtained through auxiliary vector $\mathbf{v}$, the optimal solution of $a$ and $b$ cannot be found directly. It should be noted that the modulus of the auxiliary vector $\mathbf{v}$ is always equal to 1 during optimization. This is equivalent to apply a constant modulus constraint to $\mathbf{v}$ and causes the problem (27) to non-convex.

In this section, an alternating iteration method is proposed to solve the optimization problem. Both the expected signal and the weighting coefficients are updated in the process of objective function optimization constantly.

Firstly, for given $a, b$ and $c, \mathbf{v}$ making the objective function optimal is immediate, let

$$
\mathbf{u}=\left(a \mathbf{z}_{1}+b \mathbf{z}_{2}\right) \odot(1 / c \mathbf{z})
$$

Consider constant modulus constraint on $\mathbf{v}$, the optimal

$$
\mathbf{v}=\exp (j \arg (\mathbf{u}))
$$

where $\arg (\cdot)$ denotes argument of a complex number.

Then, fix $b, c$ and $\mathbf{v}$ to optimize $a$. When these three variables are fixed, the analytic solution of the optimal $a$ can be obtained directly. Differentiate $O$ with respect to $a$

$$
\frac{\partial O}{\partial a}=a \mathbf{z}_{1}{ }^{\mathrm{H}} \mathbf{z}_{1}-\mathbf{z}_{\mathbf{1}}{ }^{\mathrm{H}}\left(c \mathbf{z} \odot \mathbf{v}-b \mathbf{z}_{2}\right)
$$

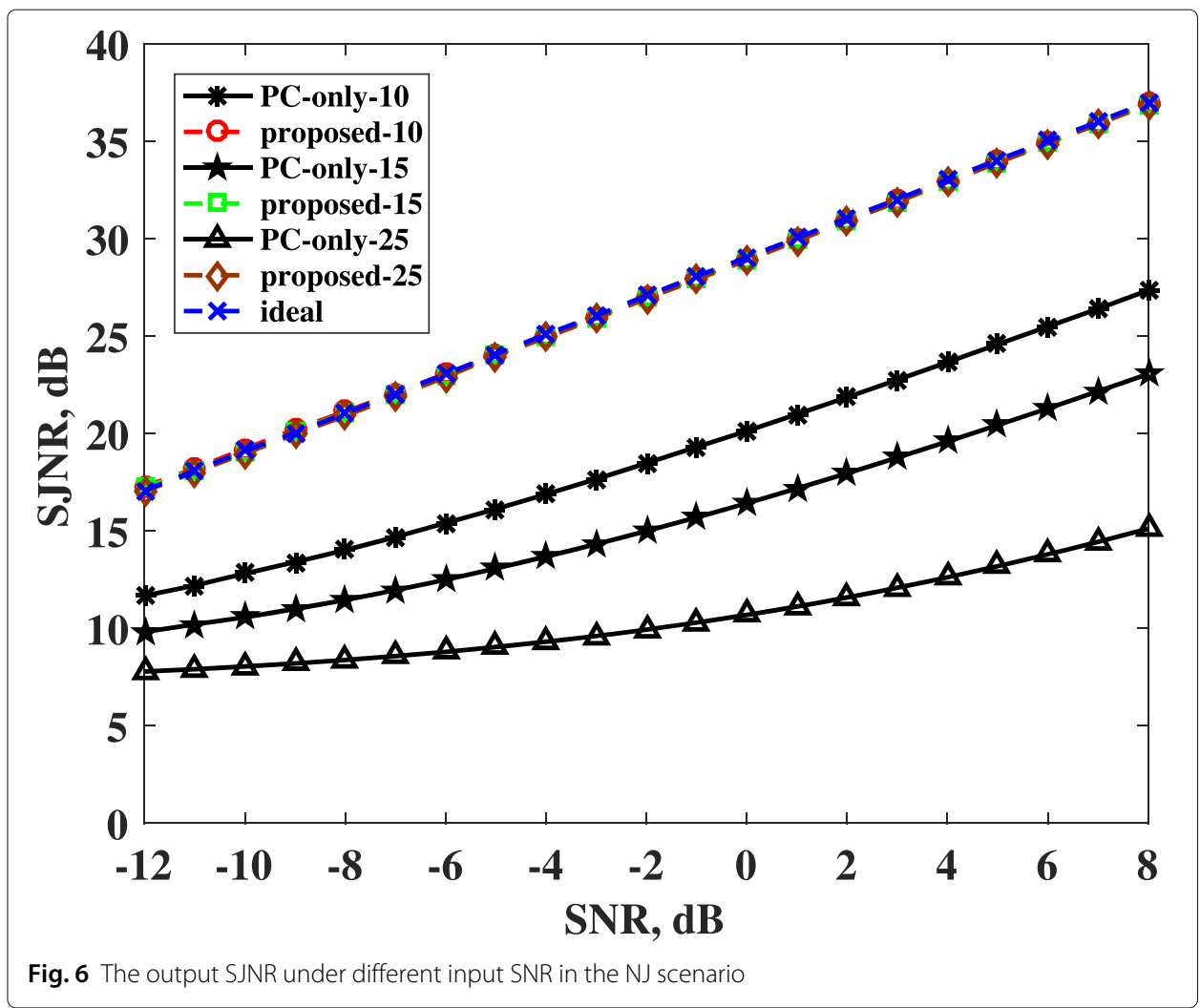


Let (30) equal to 0 to get the optimal $a$

$$
a=\frac{\mathbf{z}_{1}{ }^{\mathrm{H}}\left(c \mathbf{z} \odot \mathbf{v}-b \mathbf{z}_{2}\right)}{\mathbf{z}_{\mathbf{1}}{ }^{\mathrm{H}} \mathbf{z}_{\mathbf{1}}}
$$

Similarly, fix $a, c$ and $\mathbf{v}$ to optimize $b$

$$
b=\frac{\mathbf{z}_{2}{ }^{\mathrm{H}}\left(c \mathbf{z} \odot \mathbf{v}-a \mathbf{z}_{1}\right)}{\mathbf{z}_{2}{ }^{\mathrm{H}} \mathbf{z}_{2}}
$$

Fix $a, b$ and $\mathbf{v}$ to optimize $c$

$$
c=\frac{(\mathbf{z} \odot \mathbf{v})^{\mathrm{H}}\left(a \mathbf{z}_{1}+b \mathbf{z}_{2}\right)}{(\mathbf{z} \odot \mathbf{v})^{\mathrm{H}}(\mathbf{z} \odot \mathbf{v})}
$$

The flow for the cyclic local minimization of the MMSE metric in (27) can be summarized as follows.

Step 0: Set the $a, b$ and $c$ to some initial values;

Step 1: Compute $\mathbf{v}$ for $a, b$ and $c$ fixed [see (28), (29)];

Step 2: Compute $a$ for $b, c$ and $\mathbf{v}$ fixed [see (31)];

Step 3: Compute $b$ for $a, c$ and $\mathbf{v}$ fixed [see (32)];

Step 4: Compute $c$ for $a, b$ and $\mathbf{v}$ fixed [see (33)];

Iteration: repeat Steps 1-4 until a prespecified stop criterion is satisfied, e.g. $\left\|a^{(i)}-a^{(i+1)}\right\| \leq \varepsilon,\left\|b^{(i)}-b^{(i+1)}\right\| \leq \varepsilon, a^{(i)}, b^{(i)}$ are the values obtained at the $i$ th iteration, $\varepsilon$ is a predefined threshold, such as $10^{-6}$. In addition, if the algorithm reaches the maximum iteration number, the optimization process should stop immediately.

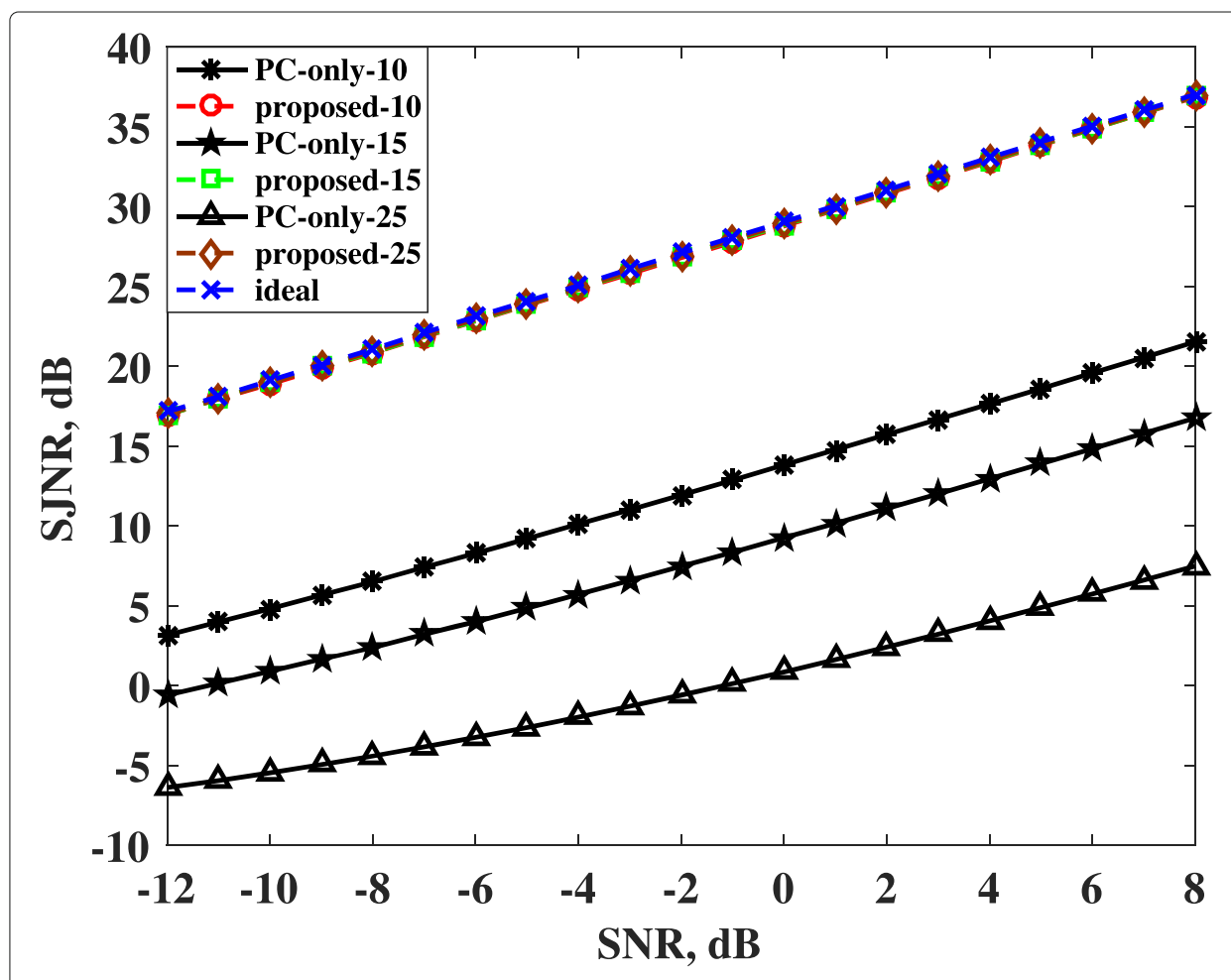

Fig. 7 The output SJNR under different input SNR in the CNJ scenario 
At the end of iteration, inverse fast Fourier transform (IFFT) operation is performed on the weighted signal to obtain the final output signal:

$$
\mathbf{y}=\mathbf{A}\left(a_{\mathrm{o}} \mathbf{z}_{1}+b_{\mathrm{o}} \mathbf{z}_{2}\right)
$$

where $a_{\mathrm{o}}, b_{\mathrm{o}}$ denote the final output of $a$ and $b$ after optimization. After IFFT operation, the signal is converted from frequency domain to time domain for subsequent target detection and parameter estimation.

\subsection{Summary}

The flow chart of the method presented in this paper is shown in Fig. 1. A LFM radar also needs to do FFT and IFFT operation on the received signal when performing conventional PC processing. The computational burden of the proposed algorithm is mainly for the iterative optimization of several variables. Only simple matrix addition and multiplication are involved.

It can be seen from (16) and (17) that the constant modulus constraint of the auxiliary vector $\mathbf{v}$ makes the recovered signal match the target feature as much as possible, while eliminating the interference signal.

For moving targets, the Doppler frequency is very critical information. It plays a vital role in subsequent target recognition and tracking. We hope to retain the Doppler information of the target while suppressing jamming. According to the previous analysis, the Doppler information in the slow-time domain is reflected in the parameter $c$ and participates in the optimization process, while fast-time domain information is ignored in

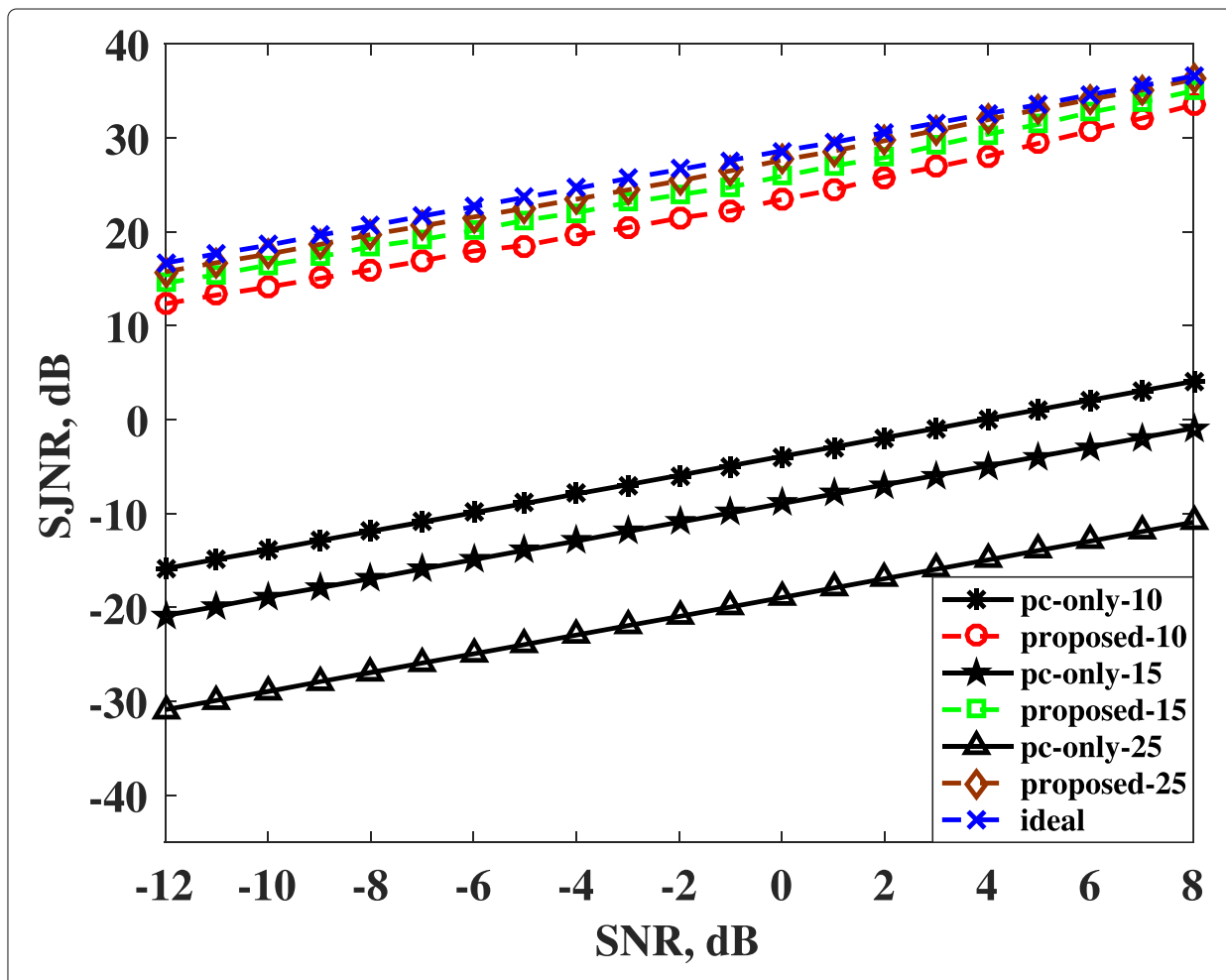

Fig. 8 The output SJNR under different input SNR in the DFTJ scenario 
the derivation. The received data contains complete fast-time domain Doppler information that is not corrupted by the product of the received data vector and a scalar. The method proposed in this paper restores the target echo without losing the Doppler information. The conclusion will be reflected in the processing results of the measured data in Section 5.

In the above derivation, we directly ignore the noise component, which is very reasonable in the scenario with high SNR or jamming-to-noise-ratio (JNR). Considering various application scenarios, we discussed the situation where noise cannot be ignored relative to the target component and the interference component. In all PRI sampling points, the target echo only occupies a part, and (21) is valid only at these points. For the points without target echo (noise or noise plus interference only), the optimization objective function in (27) is meaningless to them. These points can disrupt the target recovery process to some extent. In practice, instead of sampling all the points for processing, some points within a certain range gate are taken. This operation reduces the energy of the disturbance component and weakens the negative influence of noise on the target signal recovery.

\section{Results and Discussion}

In this section, we present numerical studies to illustrate the performance of the proposed algorithm. The performance is evaluated from two aspects. The first metric is the ability of the algorithm to suppress jamming. The second metric is the ability of the algorithm to recover the target echo.

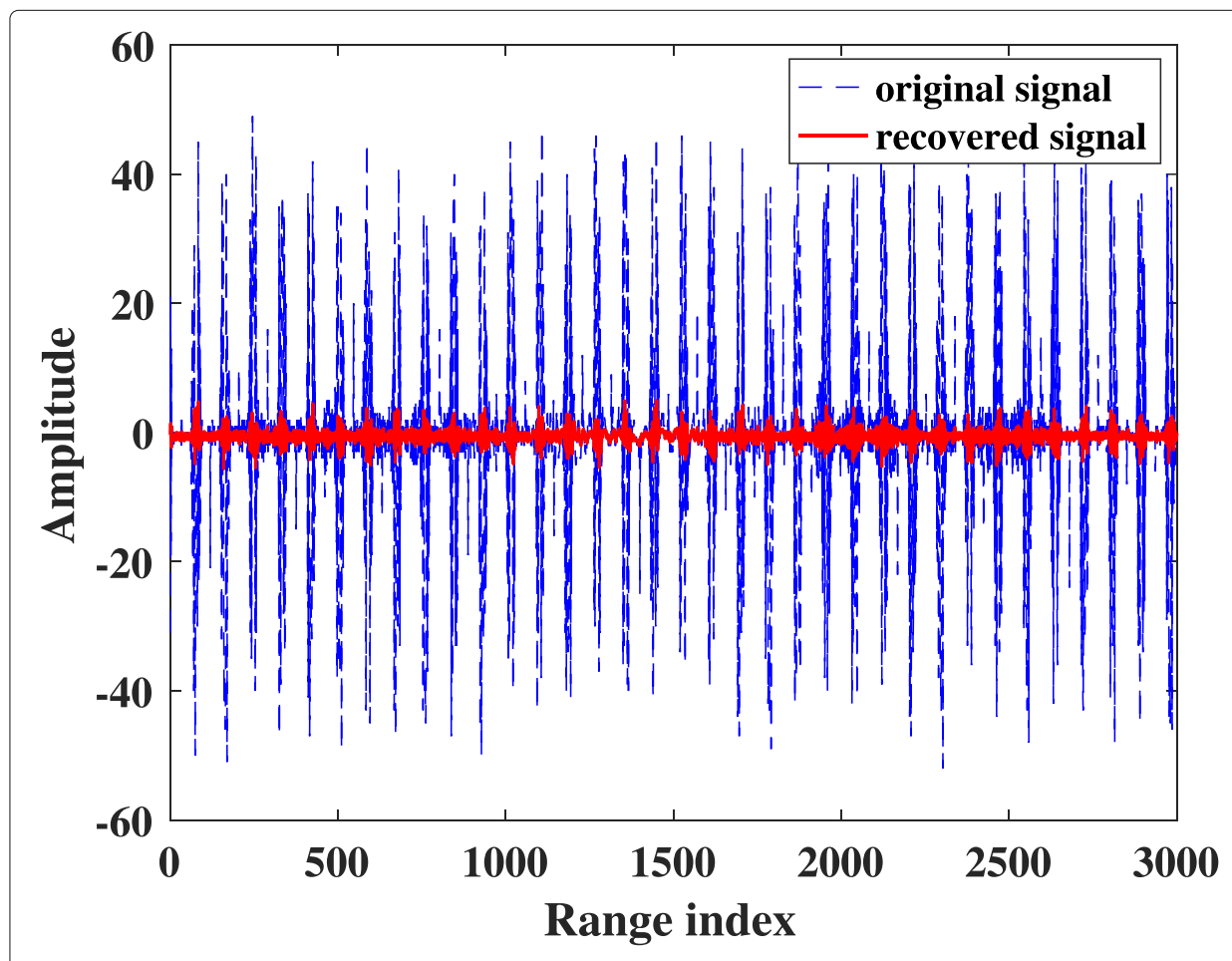

Fig. 9 Real part of the original signal and the recovered signal 


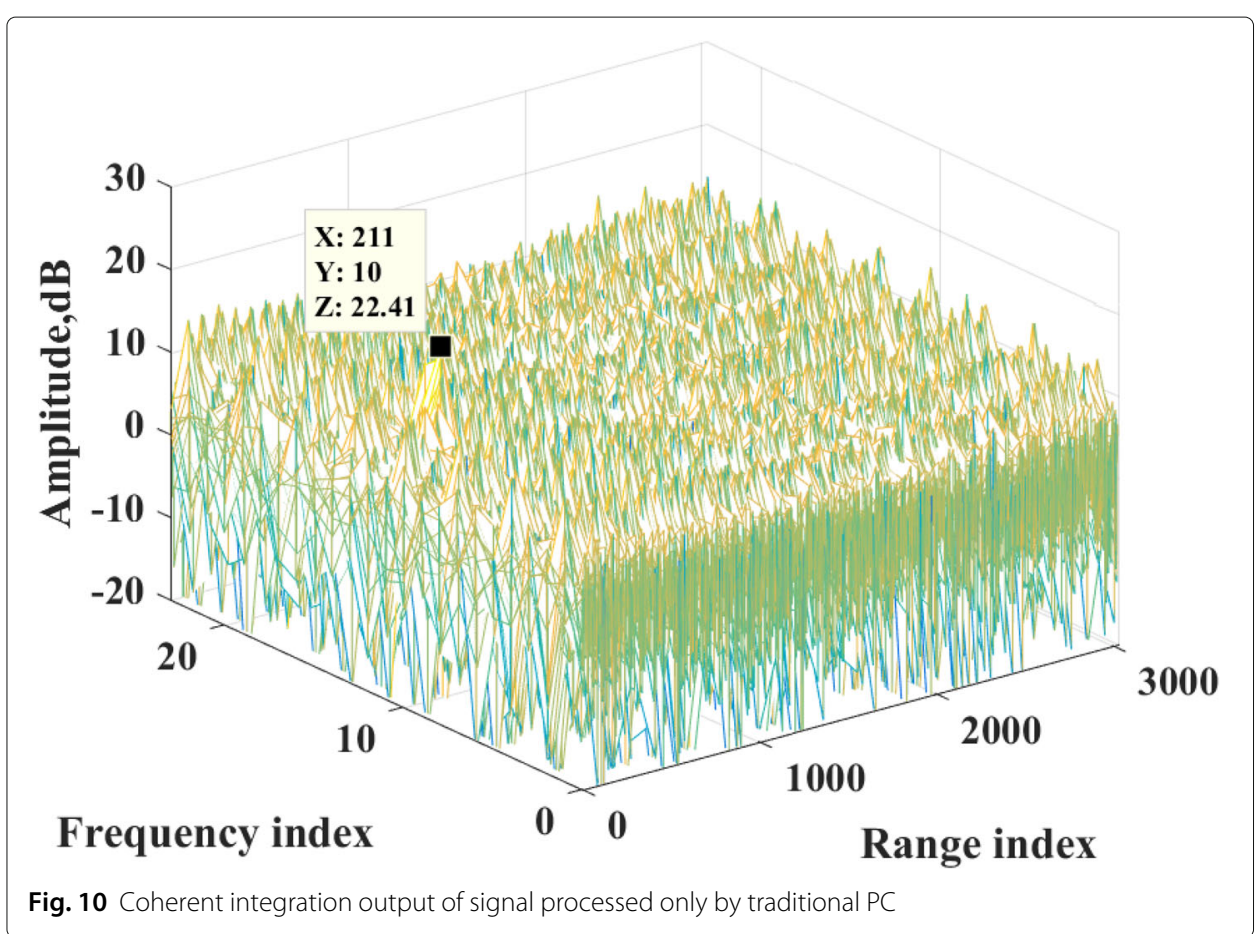

$\mathrm{NJ}$ and smart jamming are used to verify the robustness of the proposed algorithm. The characteristics of jamming signals are described as follows:

A complex Gaussian random sequence with zero mean and variance of 1 was used to simulate NJ. NJ is a kind of continuous-wave whose duration is much longer than the transmitting pulse width of radar.

Smart jamming is generally implemented in the form of a pulse, and the duration is related to the length of the convolution sequence. The simulation of smart jamming includes two scenarios. The Gaussian distribution random sequence with zero mean and variance of 1 is used to convolve with a copy of the transmitted signal stored in DFRM to generate CNJ. Affected by the difference of sampling period between jammer and radar, the other kind of smart jamming forms several false range targets at the radar receiver with long intervals. The sequence used to gain DFTJ consists of 0 and 1 . The elements 1 lie in the $[1,101,201,301,401,501,601,701]$ th indexes and 0 lie in others. The length of the sequence used to obtain CNJ and DFTJ is equal to the pulse width of the radar signal.

Assume the polarization vector of the main antenna $\mathbf{h}_{\mathrm{m}}=[1,0.05]^{\mathrm{T}}$; the polarization vector of the cross-polarization auxiliary antenna $\mathbf{h}_{\mathrm{a}}=[0.05,1]^{\mathrm{T}}$; the polarization scattering matrix of the target $\mathbf{S}_{\mathrm{p}}=\frac{1}{2}\left[\begin{array}{cc}1.0 & 0.4 \mathrm{j} \\ -0.4 \mathrm{j} & 0.9\end{array}\right]$; the polarization vector of the jammer's transmitting antenna $\mathbf{h}_{j}=\frac{1}{\sqrt{2}}[1, \mathrm{j}]^{\mathrm{T}}$; Both of the channel noise in the main channel and the auxiliary channel are modeled by complex Gaussian distribution with zero mean and variance $\sigma_{n}^{2}=1$, and independent of each other.

\subsection{Simulation for jamming suppression}

In this section, the results of traditional PC and the proposed algorithm to suppress jamming are compared. 
We set up a specific application scenario. Assume radar transmits a pulsed LFM signal, and the relevant waveform parameters are displayed as follows: the pulse duration is $10 \mu \mathrm{s}$, the bandwidth is $40 \mathrm{MHz}$, and pulse repetition period is $200 \mu \mathrm{s}$. The sampling frequency is $80 \mathrm{MHz}$.

In practical applications, the radar signal processor only does the digital sampling of signals within a certain range gate, rather than on the entire PRT. In the simulation, 1600 points near the range gate of the target are taken. For interference with a long duration, complete interference samples are not taken, which will not affect the target recovery.

Define SNR as $20 \log \left(\mathbf{h}_{\mathrm{m}}^{\mathrm{T}} \mathbf{S}_{\mathrm{p}} \mathbf{h}_{\mathrm{m}} A_{\mathrm{m}, \mathrm{x}} / \sigma_{n}^{2}\right)$, JNR as $20 \log \left(\mathbf{h}_{\mathrm{m}}^{\mathrm{T}} \mathbf{h}_{\mathrm{j}} A_{\mathrm{m}, \mathrm{j}} / \sigma_{n}^{2}\right)$. Adjust $A_{\mathrm{m}, \mathrm{x}}$ and $A_{\mathrm{m}, \mathrm{j}}$ to change the SNR and JNR (refer to SNR and JNR of the main channel unless otherwise specified), and set $A_{\mathrm{a}, \mathrm{x}}$ equal to $A_{\mathrm{m}, \mathrm{x}}$, and $A_{\mathrm{a}, \mathrm{j}}$ equal to $A_{\mathrm{m}, \mathrm{j}}$.

Assume the target is located in the 6051st range bin. NJ covers 15,000 range bins from 1001st to 16,000 th. CNJ and DFTJ located in the 6000th range bin. In this scenario, we use the proposed method to recover the target echo. The sampling process starts at the 5900th point. For comparison, we also plot the result of the ideal case which means no interference.

Set the iteration stop threshold $\xi=10^{-6}$, and the maximum iteration number is 1000 . Set the initial values of $a=1, b=1, c=1$.

Figure 2 shows the final output of the traditional PC and the proposed jamming suppression algorithm in NJ scenario. $\mathrm{NJ}$ is a kind of incoherent interference, and there is no gain in PC processing. Effective NJ has certain requirements for interference power. We set $\mathrm{SNR}$ as $5 \mathrm{~dB}$ and JNR as $20 \mathrm{~dB}$. It can be seen that before interference suppression, high-power NJ drowned the target echo, and the base noise level of radar is raised with no target output. After interference suppression, the target echo is successfully recovered. Compared with the ideal case, the range bin where the target is located remains consistent, and there is almost no SNR loss.

Figure 3 shows the results in CNJ scenario. We set SNR as $5 \mathrm{~dB}$ and JNR as $15 \mathrm{~dB}$. After $\mathrm{PC}$, the $\mathrm{CNJ}$ appears as false targets occupying continuous range bins. When the real target is located in the false target group, the radar cannot detect the real target. $\mathrm{CNJ}$ jamming plays the dual role of deception and blanket. After interference suppression, the false target group disappears, while the real target is preserved completely.

Figure 4 shows the results in DFTJ scenario. The input SNR and JNR are consistent with the CNJ scenario. After PC, DFTJ presents as multiple false targets. Each false target is located at a certain range cell, and the real target can be detected, but it is difficult to distinguish the true target from the false targets. After the interference suppression, only the real target is retained, and there is no need to identify the true or false target.

The simulation results show that the proposed method can achieve the two metrics mentioned above.

Figure 5 shows the convergence of the optimization algorithm. In the figure, 'NJ-10' represents the simulation result when JNR is $10 \mathrm{~dB}$ under the background of NJ. The other symbols have a similar meaning and are not tautological here. When JNR $=10 \mathrm{~dB}$, the algorithm reached the optimal value after 300 iterations. When JNR $=20 \mathrm{~dB}$, the algorithm stopped iteration after reaching the maximum iteration number 1000. The higher the JNR is, the slower the convergence speed is. 


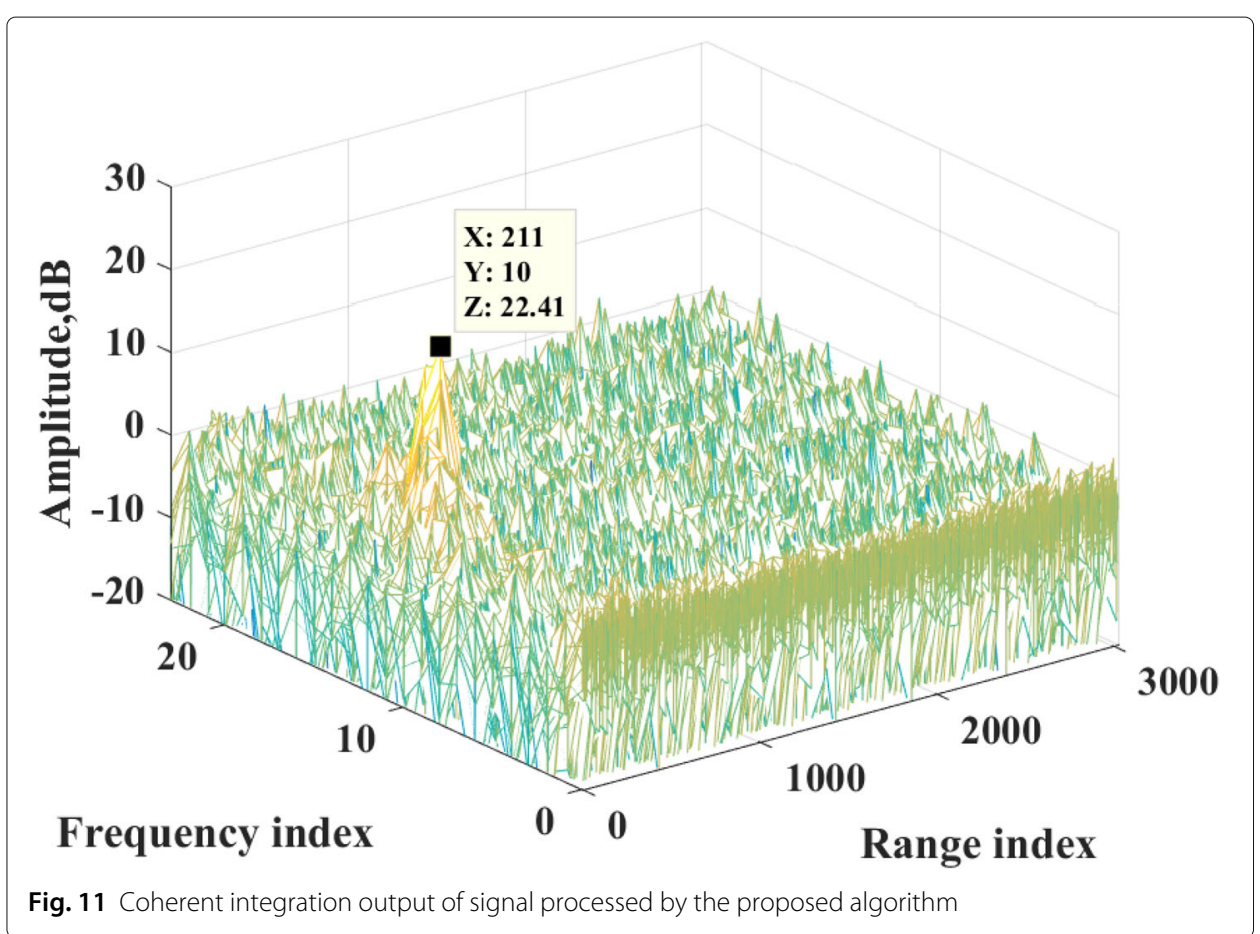

\subsection{Performance analysis}

In this section, a set of experiments are designed to show the performance of the proposed algorithm under different SNR. Adjust $A_{\mathrm{m}, \mathrm{x}}$ to change the SNR from $-12 \mathrm{~dB}$ to $8 \mathrm{~dB}$ in the scenario where JNR is $10 \mathrm{~dB}, 15 \mathrm{~dB}$, and $25 \mathrm{~dB}$, respectively, the echo was processed with the proposed method, and the signal-to-jamming and noise ratio (SJNR) before and after interference suppression was calculated as an evaluation index.

Considering the requirement for radar to process real-time data with high speed, the maximum iteration number was set as 300 in the subsequent simulation.

The final output signal in time domain is used to calculate the SJNR, the range bin where the target is located (152nd point in the output sequence) is used to calculate the signal power. For NJ, take points from 250th to 1200th in the output sequence as interference samples to calculate the power of interference and noise. For $\mathrm{CNJ}$, take points from 250th to 850th as samples. For NJ and CNJ, SJNR determines whether the target can be detected normally. For DFTJ, SNR determines whether the target is detected or not, and JNR determines whether the false target is valid. The interference power is calculated by [101, 201, 301, 401, 501, 601, 701, 801]th points, the noise power is calculated by points from 1200th to 1500th, and the maximum value of the two is taken as the power of interference plus noise to calculate SJNR. The difference between the actual SJNR and the ideal SJNR can be understood as the JNR with the residual false target. Statistical results are obtained from 500 Monte Carlo experiments.

Figures 6, 7, and 8 show how the output SJNR change with the input SNR, before and after suppression of the three kinds of interference, respectively. In the figure, "PC-only10 " represents the SJNR value of the signal processed only by traditional PC when JNR is $10 \mathrm{~dB}$, "proposed-10" represents the SJNR of the signal processed by the proposed algorithm when JNR is $10 \mathrm{~dB}$, and so on with the other symbols. It can be seen from Figs. 6 and 
7 that the output SJNR after NJ and CNJ suppression can almost reach the ideal condition without interference under different SNR and JNR. The simulation results in Fig. 8 show a 3-4 dB SNR loss after DFTJ suppression, which can be interpreted as JNR of the residual false target is $4 \mathrm{~dB}$, and valid false targets cannot be formed at the detection result. It is reasonable to think DFTJ suppression is successful. It is proved that the proposed method can suppress the three interference effectively.

For the scenario with high SNR, the 300 iterations may not achieve the optimal effect, but it can be seen from Figs. 6, 7, and 8 that the impact of this problem on the results can be completely ignored.

\subsection{Measured data}

In this section, the effectiveness of the proposed method is verified by radar measured data. The jamming in the data is NJ. The transmitted signal is LFM with a pulse duration of $24 \mu \mathrm{s}$, and bandwidth of $85 \mathrm{MHz}$. Three thousand points were collected at a sampling frequency of $100 \mathrm{MHz}$. Figure 9 shows the real part of the original and the recovered signal in one PRI. The recovered signal is the result of inverse matched filtering to the output of the proposed algorithm. Coherent integration is done with 24 pulses at the same beam position. Figures 10 and 11 show the result of coherent integration. Before and after interference suppression, the target is in the same Doppler channel, which verifies the interference suppression process does not lose Doppler information of the target. Figure 12 shows the slice of according Doppler channel, the signal processed only by PC and processed by the proposed algorithm are compared in the same axis. After interfere-

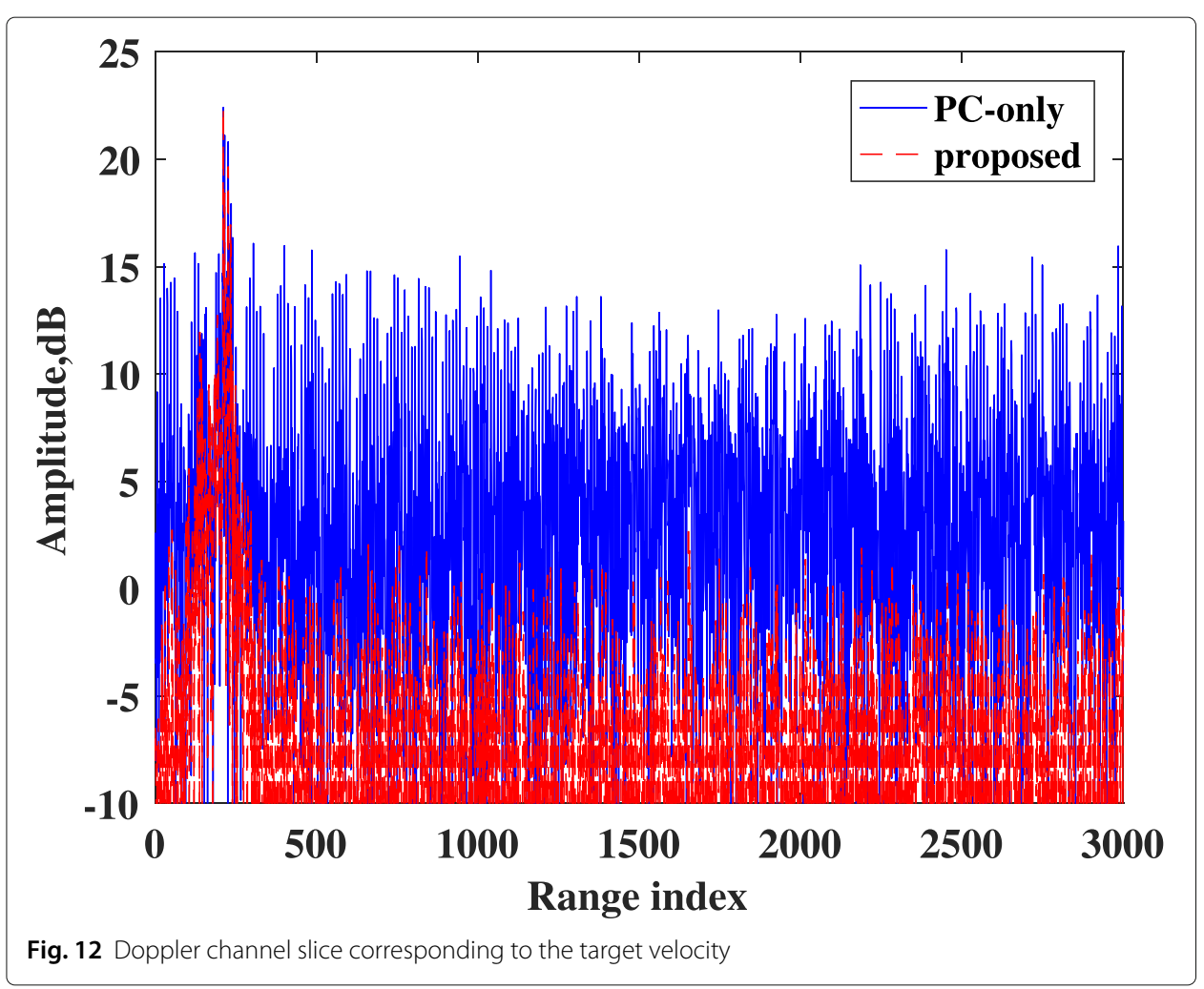


nce suppression, the SJNR gain is $13 \mathrm{~dB}$. It can be concluded from the figure that the proposed method can suppress this batch of jamming effectively.

\title{
5 Conclusion
}

In this paper, the method for suppressing $\mathrm{NJ}$ and smart jamming is studied based on radar transmitted waveform matching. The signal extraction problem is converted into an optimization problem, and the optimal weighting coefficients are obtained by optimizing the objective function iteratively. By weighting the two aliased signals in main channel and cross-polarization auxiliary channel, the target signal can be recovered successfully, which is very close to the ideal performance in the non-interference scenario. The proposed method can suppress the jamming and retain the real target signal component simultaneously, overcome the disadvantage of poor versatility of the suppression method and reduce the adverse effects caused by the loss of echo information during the suppression process. The results of the measured data indicate that the method is effective in practice. The optimization algorithm only needs about 300 iterations to achieve a good interference suppression effect and will not bring too much computational burden for radar signal processing.

It should be concerned that due to the limitation of the number of independent observation channels, this method can only cope with one interference source. However, the proposed idea of constructing a reference signal based on the radar transmitted signal can be transferred to a multi-channel scenario such as a digital array radar to deal with multiple interference sources.

\begin{abstract}
Abbreviations
NJ: Noise jamming; SNR: Signal-to-noise ratio; PC: Pulse compression; RCS: Radar cross-section; MMSE: Minimum mean square error; RF: Radio frequency; DRFM: Digital radio frequency memory; CNJ: Convolution noise jamming; DFTJ: Dense false target jamming; LFM: Linear frequency modulated; CPI: Correlation processing interval; PRI: Pulse repetition interval; FFT: Fast Fourier transform; IFFT: Inverse fast Fourier transform; JNR: Jamming-to-noise ratio; SJNR: Signal-to-jamming and noise ratio.
\end{abstract}

Acknowledgements

Not applicable.

Authors' contributions

Xiaoying Chen found the idea and wrote this article and Baixiao Chen put forward some constructive suggestions for revision. All authors read and approved the final manuscript.

Funding

This work was supported by the National Defense Science and Technology Foundation Strengthening Program (2019-JCJQ-ZD-067-00).

Availability of data and materials

Not applicable.

\section{Declarations}

Ethics approval and consent to participate

Not applicable.

Consent for publication

Not applicable.

Competing interests

The authors declare that they have no competing interests.

Received: 28 April 2021 Accepted: 22 July 2021

Published online: 05 August 2021 


\section{References}

1. N. Li, Y. Zhang, A survey of radar ECM and ECCM. IEEE Trans. Aeros. Electron. Syst. 31(3), 1110-1120 (1995)

2. D. C. Schleher, Electronic Warfare in the Information Age. (Artech House, the Unites States, 1999)

3. D. Orlando, A novel noise jamming detection algorithm for radar applications. IEEE Signal Process. Lett. 24(2), 206-210 (2017)

4. V. Carotenuto, A. De Maio, D. Orlando, L. Pallotta, Adaptive radar detection using two sets of training data. IEEE Trans. Signal Process. 66(7), 1791-1801 (2018)

5. A. Aubry, V. Carotenuto, A. De Maio, D. Orlando, Coincidence of maximal invariants for two adaptive radar detection problems. IEEE Signal Process. Lett. 23(9), 1193-1196 (2016)

6. A. De Maio, D. Orlando, Adaptive radar detection of a subspace signal embedded in subspace structured plus gaussian interference via invariance. IEEE Trans. Signal Process. 64(8), 2156-2167 (2016)

7. S. Ahmed, Product-based pulse integration to combat noise jamming. IEEE Trans. Aeros. Electron. Syst. 50(3), 2109-2115 (2014)

8. S. Ahmed, Novel noncoherent radar pulse integration to combat noise jamming. IEEE Trans. Aeros. Electron. Syst. $\mathbf{5 1}(3), 2350-2359(2015)$

9. M. Sun, B. Tang, Noise amplitude modulation jamming signal suppression based on weighted-matching pursuit. J. Syst. Eng. Electron. 20(5), 962-967 (2009)

10. D. Du, B. Tang, A high performance algorithm of noise amplitude modulation interference suppression based on frequency domain cancellation. J. Electron. Inf. Technol. 29(3), 557-559 (2007)

11. X. Dai, H. Li, J. Ren, N. Wang, Parameters estimation of noise amplitude modulation signal. IET Radar, Sonar Navigat. 11(1), 161-170 (2016)

12. S. Guo, W. Jun, G. Chen, J. Wang, Mainlobe interference suppression based on independent component analysis in passive bistatic radar. IET Signal Process. 12(9), 1193-1201 (2018)

13. M. Ge, G. Cui, X. Yu, D. Huang, L. Kong, in 2018 IEEE Radar Conference (RadarConf18), Mainlobe jamming suppression via blind source separation (IEEE, Oklahoma City, 2018), pp. 0914-0918

14. J. Ren, P. Wang, Novel smart noise jamming suppression method based on smeared spectrum. Prog. Electromagn. Res. Lett. 67, 81-88 (2017)

15. B. Han, X. Yang, X. Wu, S. Li, Smart noise jamming suppression method based on fast fractional filtering. J. Eng. 2019(19), 6201-6205 (2019)

16. Z. Zhang, Y. Wu, J. Ren, K. Dong, in 2017 3rd IEEE International Conference on Computer and Communications (ICCC), Smart noise jamming suppression by using atomic decomposition, (2017), pp. 1377-1380

17. W. Yu, W. Chen, Smart noise jamming suppression technique based on blind source separation. Int. J. Sig. Process. Syst. 7(1), 14-19 (2019)

18. W. Liu, J. Liu, C. Hao, Y. Gao, Y.-L. Wang, Multichannel adaptive signal detection: Basic theory and literature review. Sci China Inf Sci (2021). https://doi.org/10.1007/s11432-020-3211-8

19. L. Griffiths, C. Jim, An alternative approach to linearly constrained adaptive beamforming. IEEE Trans. Antennas Propag. 30(1), 27-34 (1982)

\section{Publisher's Note}

Springer Nature remains neutral with regard to jurisdictional claims in published maps and institutional affiliations.

\section{Submit your manuscript to a SpringerOpen ${ }^{\circ}$ journal and benefit from:}

- Convenient online submission

Rigorous peer review

- Open access: articles freely available online

- High visibility within the field

- Retaining the copyright to your article

Submit your next manuscript at $\boldsymbol{\nabla}$ springeropen.com 